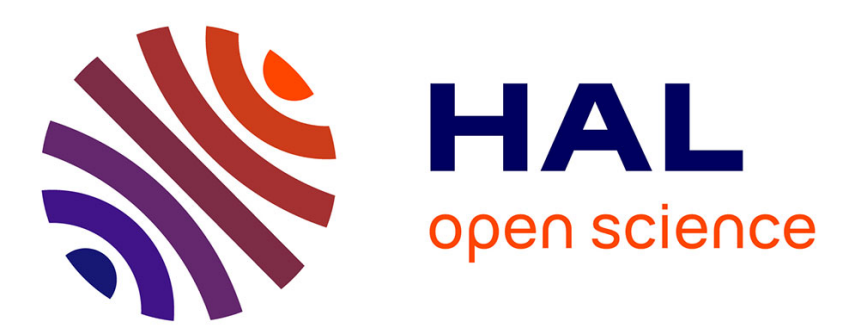

\title{
Using the cognitive interview to recall real-world emotionally stressful experiences: Road accidents
}

Olivier Dodier, Magali Ginet, Frédérique Teissedre, Fanny Verkampt, Ronald Fisher

\section{- To cite this version:}

Olivier Dodier, Magali Ginet, Frédérique Teissedre, Fanny Verkampt, Ronald Fisher. Using the cognitive interview to recall real-world emotionally stressful experiences: Road accidents. Applied Cognitive Psychology, 2021, 35 (4), pp.1099-1105. 10.1002/acp.3828 . hal-03395955

\section{HAL Id: hal-03395955 https://hal.science/hal-03395955}

Submitted on 18 Nov 2021

HAL is a multi-disciplinary open access archive for the deposit and dissemination of scientific research documents, whether they are published or not. The documents may come from teaching and research institutions in France or abroad, or from public or private research centers.
L'archive ouverte pluridisciplinaire HAL, est destinée au dépôt et à la diffusion de documents scientifiques de niveau recherche, publiés ou non, émanant des établissements d'enseignement et de recherche français ou étrangers, des laboratoires publics ou privés. 


\section{Using the Cognitive Interview to Recall Real-world Emotionally Stressful Experiences:}

\section{Road Accidents}

Olivier Dodier ${ }^{1}$, Magali Ginet ${ }^{2}$, Frédérique Teissedre ${ }^{2}$, Fanny Verkampt $^{3}$, \& Ronald P. Fisher ${ }^{4}$

${ }^{1}$ Faculté de Psychologie, Université de Nantes, France

${ }^{2}$ Laboratoire de Psychologie Sociale et Cognitive (UMR CNRS 6024), Université Clermont Auvergne, Clermont-Ferrand, France

${ }^{3}$ CLLE, Université de Toulouse, CNRS, UT2J, France

${ }^{4}$ Florida International University, Miami, USA

Manuscript in press in Applied Cognitive Psychology

Corresponding authors:

Olivier Dodier, Faculté de psychologie, Université de Nantes, Chemin de la Censive du Tertre, Nantes Cedex 3, France, E-mail address: ododier.univ@gmail.com

Magali Ginet, Université Clermont Auvergne, CNRS, LAPSCO, 34 Avenue Carnot, F-63000, Clermont-Ferrand, France, E-mail address: magali.ginet@uca.fr

Conflict of Interest:

The authors declare no conflict of interest in relation to this manuscript.

Data availability statement:

The data that support the findings of this study are available on request from the corresponding authors. The data are not publicly available due to privacy or ethical restriction. 


\begin{abstract}
This study examined the relevance of the cognitive interview (CI) for the recall of a road accident by actual victims, depending on the level of stress experienced at the time of the event. Fifty-six victims (or witnesses) were interviewed, either with a CI or control interview, after completing a series of questionnaires including measures of their stress level during the accident. Participants who reported a high level of stress recalled more central details than those with a low level. In addition, participants interviewed with the CI recalled more details than those interviewed with a control interview, regardless of the level of stress experienced during the accident. This experiment confirms the CI's relevance in a stressful context, and after an extended period of time. Practical implications for interviewing victims are discussed. Keywords: eyewitness testimony, cognitive interview, mental context-reinstatement, stress, investigative interviewing
\end{abstract}


Because memory is fallible and sensitive to a wide variety of factors (e.g., stress), interview methods have been developed to safeguard as much as possible the reliability of witnesses' memories. Fisher and Geiselman (1992) therefore developed the cognitive interview (CI) to enhance the completeness of recalls. The CI is a non-suggestive and witness-centred interview protocol that includes communication rules and social dynamics, such as establishing rapport, encouraging active witness participation or promoting extensive and detailed responses. It also contains some memory-enhancing components, such as mental context reinstatement (mentally recreating the environmental and personal context of the event). Many research studies have highlighted the forensic value of this protocol. CI recalls are more complete, and despite a small increase in the number of errors, no decrease in accuracy rate (i.e., the ratio of correct information to total information recalled) is observed compared to control interviews (suggesting an increase in the number of correct details reported; see Memon, Meissner \& Fraser, 2010). However, only a few studies have been conducted to assess the benefit of the CI under conditions of high stress, with actual witnesses (Colomb et al., 2013; Clifford \& George, 1996; Fisher, Geiselman, \& Amador, 1989; Peace \& Porter, 2004).

Studies assessing the effects of acute stress on eyewitness memory have shown that emotional stress enhances the retrieval of central details of an event, while impairing the retrieval of peripheral details (Christianson, 1992). Central information is "information that is connected with the source of the emotional arousal (the gist of the event and its central details)" whereas peripheral information is "information that is irrelevant or spatially peripheral to the source of the emotional arousal (peripheral details)" (Christianson, 1992, p. 291). These definitions are consistent with a meta-analysis of the effect of acute stress on episodic memory, which concluded that the stress felt during encoding improves memory 
performance when the items to be retrieved are stressor-relevant (vs. non-stressor-relevant items; Shields et al., 2017).

In these different studies, emotional stress is viewed as affecting memory mainly during encoding. However, stress experienced during encoding can also affect retrieval processes (Shields et al., 2017). More specifically, stress could be part of the internal context in the same way as mood in the mood-dependent memory (MDM) literature (Eich, 1995). In line with MDM, if stress is not reinstated during the retrieval stage, the internal context is then different between encoding and retrieval contexts, leading to a possible decrease in recall performances. Reinstating, for example mentally, the internal context during retrieval should then improve recall, especially of the details most affected by stress (peripheral details). In fact, mental context reinstatement is a core retrieval instruction of the CI.

There is a lack of data regarding the relevance of the $\mathrm{CI}$ in situations of very high stress or trauma (Memon et al., 2010). Some laboratory studies have shown that the CI or techniques based on the CI (e.g., Self-Administered Interview) were effective, regardless of the level of stress experienced during encoding (Ginet \& Verkampt, 2007; Krix et al., 2016). However, the levels of stress manipulated were likely to be very moderate compared to those experienced in real stressful situations.

Other studies demonstrated that the CI was effective in the field with actual witnesses and victims of crime who may have experienced high levels of stress (Clifford \& George, 1996; Fisher et al., 1989 ; Colomb et al., 2013) or with participants who had experienced a traumatic event (Peace \& Porter, 2004). However, in these studies, stress was not measured directly with the victims, making it impossible to assess the benefit of the $\mathrm{CI}$ at different levels of stress.

In the current study, 56 low-stressed or high-stressed victims of traffic road accidents were interviewed with either a CI or a control interview protocol with the aims to determine 
the influence of the CI on the quantity of details recalled (overall quantity and number of central and peripheral details). Traffic road accidents were chosen because they are frequent ${ }^{1}$, may require law enforcement intervention, and are a type of stressful event that has not been field tested with the CI to date. In light of the theoretical considerations, we expect that: (i) The CI (vs. control) will improve the quantity of reported information (irrespective of the centrality); (ii) Participants in the high stress condition (vs. low) will recall more central details, but fewer peripheral details; (iii) The deleterious effect of a high level of stress on the recall of peripheral details should be observed particularly with the control interview, less with the CI.

\section{Method}

\section{Design}

This study has a 2(interview type: CI, control) $\times 2$ (stress: high, low) between-subject factorial design. Participants were assigned randomly to the CI $(n=29)$ or control interview ( $n=27)$ conditions. Several dependent variables were used: (i) event-related details (total, central and peripheral details), and (ii) subjective details (for reasons of brevity, see Supplemental Material Online for this variable).

\section{Participants}

Participants characteristics and sample size. Initially, 67 people agreed to participate. Eleven participants were excluded because of: too high score $(>16)$ on the Beck Depression Inventory (Beck, Steer, \& Garbin, 1988) and/or on the Impact of Event Scale (Sundin \& Horowitz, 2002; > 42) $(n=3)$, brain injury-related amnesia $(n=2)$, absence at the recall phase $(n=2)$, technical problems during interview recording $(n=2)$, weak mastery of the French language $(n=1)$, and lack of cooperation during the recall phase $(n=1)$. In total,

\footnotetext{
${ }^{1}$ In France, in 2018, 58,352 road accidents occurred, resulting in 3,488 deaths and 7,325 injuries. See https://www.securite-routiere.gouv.fr/la-securite-routiere-en-chiffres-lobservatoire-national-interministeriel
} 
56 participants took part in the study. The sample was composed of 16 males $\left(M_{\mathrm{age}}=21.5\right.$; $\left.S D_{\text {age }}=3.4\right)$ and 40 females $\left(M_{\text {age }}=20.3 ; S D_{\text {age }}=1.8\right)$.

The interview occurred on average 10.29 months after the accident $(S D=6.90$; range $=0-25$ months $)$. The mean duration between the accident and the interview did not differ between the two interview protocol conditions (CI: $M=9.37 ; S D=6.05 ;$ range $=2-21$ months; control interview: $M=10.96 ; S D=7.35$; range $=0-25$ months), $t(52)=0.87, p=.39$, $d=0.24$. Non-student participants $(n=3)$ received $20 €$, and student participants $(n=53)$ received course credits.

Recruitment. Posters were displayed in the university and flyers were distributed, both inviting people who had experienced a road accident in the 2 previous years to participate in the study. Inclusion criteria were: being over 18 years old, having experienced a road traffic accident as a victim within the two past years, but without any deaths in the accident.

Nature of the road accidents. Among the participants, $44.6 \%(n=25)$ testified about run-off-road or head-to-tail collisions, 39.3\% $(n=22)$ about a collision with another vehicle (car or two-wheelers), 5.4\% $(n=3)$ about collisions involving pedestrians, $3.6 \%(n=2)$ about a collision with large animals, and $1.8 \%(n=1)$ about a fire in a bus. Participants reported that they $(35.7 \%, n=20)$ or other people $(25 \%, n=20)$ suffered physical injuries as a result of the accident.

\section{Material}

Self-administered Emotional Stress Scale. For the purposes of the study, we created a 6-item scale based on the one used by Ginet and Verkampt (2007), and on Christianson's (1992) definition of a negative emotional event. The scale allowed us to evaluate the level of stress (high vs. low) experienced by the participants during the encoding process. It consisted of six items (stress, displeasure, surprise, arousal, physiological changes, personal threat) with 
a 7-point Likert scale ranging from 1 (not at all) to 7 (extremely), resulting in a minimum total score of 6 and a maximum score of 42 . The internal consistency of the 6 items was satisfactory (Cronbach's $\alpha=0.75$ ). Total score (based on scores to the 6 items) was thus used as an independent inter-subject variable "Level of stress, " with 2 conditions (high, low) determined by a median split as follow: Participants with a total score $>31$ were classified into the "high level of stress" condition $(n=28)$; participants with a total score $\leq 31$ were classified into the "low level of stress" condition $(n=28)$. The median-split was motivated by the fact that preliminary analyses of the correlation between stress level and recall of central information did not show a linear relationship between these two variables $(r=-0.01, p=$ .950 , see Supplemental Online Figure).

\section{Interviews}

We recorded all interviews, with the prior permission of the participants. The mean length of the interviews was 35.5 minutes $\left(S D_{\text {lengthCI }}=15.3\right.$; range $\left.=14-75\right)$ in the CI, and 14.6 minutes $\left(S D_{\text {length control interview }}=10.6\right.$; range $\left.=3-45\right)$ in the control interview.

The cognitive interview. We used the latest version of the Cognitive Interview (Geiselman \& Fisher, 2014). The interview began with establishing a trust-based relationship between interviewers and participants, by asking non-invasive personal questions (e.g., hobbies). Then, interviewers explained to participants that the purpose of the interview was to help them remember better and to collect as much detail as possible. The next step referred to the transfer of control of the interview: Participants were told that they will do most of the talking and that only they were able to provide details about the event. Then, interviewers presented the report-everything (recall as many details as possible, even if they seem insignificant or incomplete) and no-guessing (do not guess or invent) instructions. Interviewers also informed the participants of the possibility of taking a break whenever they wished. Then, participants were encouraged to say when they did not understand something 
or when they did not know. They were invited to make a sketch to clarify their recall. After this introductory phase, interviewers invited participants to mentally reinstate the external and internal context in which they encoded the event. Interviewers then invited the participants to recall the traffic road accident as soon as they felt ready to do so. There was no time limit for free recall, which stopped when participants clearly expressed that they had nothing more to recall (e.g., "can you recall anything else?" - "that's all I can remember"). It was made clear that it was a free recall task and that there were no particular constraints. Once the first free recall ended, interviewers asked follow-up questions to deepen the details reported by the participants, using the witness-compatible questioning technique. Interviewers repeated the social and communication rules (report-everything instruction, the possibility to say "I don't know" and to draw a sketch, etc.) throughout the interview.

The Control Interview Protocol. This protocol was a bare-bones interview that included the basic elements of proper interviewing, but without the communication-enhancing and retrieval-enhancing CI elements. However, we retained an open-ended narration so that we could compare recall performance under the two conditions.

The control interview consisted of a brief introduction of the interviewer, followed by an invitation to perform an open-ended narrative, followed by a questioning phase. Specifically, interviewers introduced themselves by their first name and explained that they wanted to help the interviewee remember better. This instruction was stated in order to control a possible motivational effect in the cognitive interview condition. Then they requested an open-ended narration. As in the CI, there was no time limit for free recall, which stopped when participants clearly expressed that they had nothing more to recall. Again, it was made clear that it was a free recall task and that there were no particular constraints. Once the recall was completed, interviewers asked follow-up questions to deepen the details reported by the participants during the open-ended narration. 


\section{Interviewers}

Eight interviewers (6 females; 2 males) conducted both interviews (CI; control interview) and administered the scales. They were all graduate student research assistants. They were $21-24$ years old $\left(M_{\text {age }}=22.5 ; S D_{\text {age }}=1.07\right)$. They all followed three 6-hours training sessions during which they were trained in the CI and the control interview. Interviewers were given a written protocol and were told to learn it. They were instructed to follow it as closely as possible during the study.

\section{Procedure}

Prior to the interview. Participants were warned that they would have to complete questionnaires and be interviewed. Then, they received by mail a pre-interview explanation sheet, the informed consent, and the pre-interview questionnaires (BDI, IES, self-reported emotional stress scale; order counterbalanced), which they had to complete one day before the interview.

Interview day. Participants gave us the completed questionnaires and we checked their BDI and IES scores. Participants with too high scores on BDI and/or IES scales were excluded at this stage and were debriefed (they received course credits or 20 euros). Then, all participants were interviewed, either with a CI or a control interview. Before leaving, participants were asked how they were feeling at the time. If the person felt a little upset, the experimenter spent a short time talking to them and let them leave only after checking that everything was fine.

\section{Coding}

The interviews' audio-recordings were transcribed verbatim and randomly coded by one of the 9 trained research assistants blinded to the experimental conditions. Coders used a coding grid, in which the free recalls and questions were separated so that separate data 
analyses could be performed. For reasons of brevity and considering the large variation in the number of questions asked $(M=14.0, S D=7.5$, range $=2-37)$, only the results on free recalls are presented in this study.

All the reported details were coded, with one point being given for each unit, and with only new information being coded (repeated information was not scored). Information was coded in three categories: event-related details, centrality of details, subjective details (see the Supplemental Material Online for this category).

Event-related details. Traditional measures in CI studies related to the description of the event itself. They included actions-, people-, location-, objects-, and conversations-related details. Given the specific nature of road accidents, and the fact that these were events that were actually experienced by the participants, we added 3 additional categories: (i) cars and vehicles, (ii) injuries, and (iii) other details (event temporal context, autobiographical elements surrounding the accident, estimates of distance, speed and duration).

Centrality of details. Reported details were also coded as central or peripheral. A detail was defined as central if "(1) it was related to what was shocking to the person in the to-be-remembered event and if (2) it could not be left out or replaced without a major change in the content of the event. Otherwise, it was classified as peripheral" (Bernsten, 2002, p. 1012).

Two independent coders coded $10.7 \%(n=6)$ of the interviews. Reliability of coding was assessed using intraclass correlation coefficients (ICC). The results for the event-related details $(\mathrm{ICC}=.95 ; 95 \% \mathrm{CI}=.68-.99)$, the central details $(\mathrm{ICC}=.97 ; 95 \% \mathrm{CI}=.82-1.0)$, and the peripheral details ( $\mathrm{ICC}=.90 ; 95 \% \mathrm{CI}=.47-.99)$ suggested excellent inter-rater reliability.

\section{Ethics committee approval}


A national ethics committee approved the research, with the recommendation not to include participants obtaining too high BDI and IES scores or involved in fatal traffic road accidents.

\section{Results}

\section{Preliminary analyses}

Three ANOVAs did not show any significant main effect of the interviewers on the total number of event-related details, $F(7,48)=1.299, p=.271, \eta_{\mathrm{p}}{ }^{2}=.096$, total number of central details, $F(7,48)=1.419, p=.220, \eta_{\mathrm{p}}^{2}=.171$, and total number of peripheral details, $F(7,48)=0.537, p=.802, \eta_{\mathrm{p}}^{2}=.073$. Furthermore, interviewer did not interact with Interview Type and $\operatorname{Stress}^{2}$ (all $F \mathrm{~s} \leq 1.45, p \mathrm{~s} \geq .23$ ). Therefore, the "Interviewer" variable was not included in any of the subsequent reported statistical tests, and we collapsed the data across interviewers in further analyses.

The number of physically injured participants did not differ according to the type of interview $\left(\chi^{2}(1, N=56)=2.176, p=.140\right.$, Cramer's $\left.V=0.197\right)$ or the level of stress $\left(\chi^{2}(1, N\right.$ $=56)=1.24, p=.265, V=0.149)$. The same applies to the number of accidents involving injured persons other than the participant (type of interview: $\chi^{2}(1, N=56)=0.02, p=.877, V$ $=0.021 ;$ stress level: $\left.\chi^{2}(1, N=56)=1.52, p=.217, V=0.165\right)$.

Finally, the total number of event-related details, central details and peripheral details was unrelated to the time delay between the accident and the recall, with respectively, $r=-$ $.074, p=.594, r=-.070, p=.614$, and $r=-.020, p=.888$.

All means, standard deviations, $F$-values, $p$-values and effect sizes can be found in Tables 1 and 2.

\footnotetext{
${ }^{2}$ The Investigator X Interview X Stress interaction effect could not be assessed because some interviewers were underrepresented in certain conditions
} 


\section{Impact of stress and interview type on recall performance}

An ANOVA was conducted to study the main and interaction effects of the type of interview used and the level of stress on the number of event-related details reported. When several dependent variables were taken into account, a MANOVA (followed by specific ANOVAs) was conducted in order to control for inflation of the type I error. This was the case for the number of details reported from each category (actions, people, location, objects, conversations, cars, injuries and other details), and for the number of central and peripheral details reported. For reasons of brevity, results on subjective details are presented in the Supplemental Material Online.

\section{Event-related details}

All results concerning the event-related details are displayed in Table 1.

A main effect of Interview Type was observed on the number of event-related details recalled. Participants in the CI condition reported almost three times as many event-related details as did participant in the control interview condition. No main effect of stress or Interview Type $\times$ Stress was observed on this measure.

Considering the category of details reported, compared to the participants in the control interview condition, the CI participants reported significantly more: actions, location details, objects, conversations, descriptions of cars, and other details. There was not a significant main effect of Interview Type on details related to people. There was also no main effect of Stress or Interview Type $\times$ Stress interaction on any of these measures, $F_{\mathrm{S}} \leq 2.545$, $p s \geq .117, \eta_{\mathrm{p}}^{2} \leq .044$.

Regarding the number of details related to injuries, participants in the CI condition reported more details related to injuries than did participant in the control interview condition. In the same vein, participants in the high-stress condition reported more details related to 
injuries than did participants in the low-stress condition. These main effects were qualified by a significant Interview Type $\times$ Stress interaction, $F(3,52)=7.556, p=.008, \eta_{\mathrm{p}}{ }^{2}=.127$. In the high-stress condition, the participants interviewed with the CI recalled more injury-related details $(M=7.93 ; S D=6.77)$ than the participants interviewed with the control interview $(M$ $=0.54 ; S D=1.13), t(26)=4.201, p<.001, d=1.52$. In the low stress condition, the

effectiveness of the CI was also significant, but slightly lower magnitude (CI: $M=2.64 ; S D=$ 2.41; control interview: $M=0.29 ; S D=0.73), t(26)=3.510, p=.003, d=1.32$.

\section{Table 1}

\section{Centrality of event-related details}

All results concerning the centrality of event-related details are displayed in Table 2.

A significant main effect of Interview Type was observed on the number of central and peripheral details recalled. Participants in the CI condition recalled significantly more central details and more peripheral details than the participants in the control interview condition. Furthermore, a significant main effect of Stress was observed on the number of central details recalled. Participants in the high-stress condition recalled significantly more central details than the participants in the low-stress condition. No significant main effect of Stress was observed on the number of peripheral details reported. No significant Interview Type $\times$ Stress interactions were found on central details and on peripheral details, $F \mathrm{~s} \leq 3.650$, $p s \geq .062, \eta_{\mathrm{p}}^{2} \leq .068$

Table 2

\section{Discussion}

Victims interviewed with the CI (compared to control interview) reported more details, in particular, more descriptions of the actions, locations, objects, conversations and 
cars, regardless of the level of stress they experienced at the time of the accident.

Surprisingly, such a benefit was not observed for describing people, which contradicts the results of several studies in the literature (e.g., Bensi et al., 2011; Holliday et al., 2012). A possible explanation is that in the road accidents investigated in the present study, and likely in many real-world car accidents, the other people are available (e.g., other passengers in the car) and are often familiar to the witness. The description of people in this case may be perceived as less necessary by witnesses. By comparison, in almost all of the other research studies showing a CI advantage when describing people, the context of the interview was a criminal investigation where, obviously, the perpetrator was unknown to witnesses and likely fled the scene. Finally, the CI elicited more central details than did the control interview, but also more peripheral details. This shows that the CI does not just lead to an increase in the recall of unimportant and useless details, but makes it possible to promote the recall of all types of details, including important and central information.

Participants who reported the highest levels of stress recalled more central information than those who reported the lowest levels. They also recalled more injury-related details. Injuries are probably the most central information for victims, and likely also the most stressful. These results are therefore consistent with the literature (e.g., Shields et al., 2017). For some authors (e.g., Levine \& Edelstein, 2009), the recall of central information depends on currently active individuals' goals (e.g., escape). More precisely, pre-goal emotions (emotions experienced before goal achievement or failure, e.g., fear), narrow the scope of people's attention to information that is central to their goals and thus enhance memory of this information. Concerning injury details, an alternative explanation could be that the most stressed (vs. the least stressed) victims were also those who suffered the most serious and complex injuries. However, we did not observe any differences in the frequency of reported injuries between the two stress conditions. 
We did not observe any interaction effects between stress and interview type on the recall of central or peripheral information. Specifically, we expected that stress would have a negative effect on recall of peripheral details and that this negative effect would be reduced in the cognitive interview condition, but we did not observe such a deleterious effect of the level of stress. Nevertheless, participants recalled more injury details when they were interviewed with the CI (vs. control interview), and even more so when they experienced high (vs. low) levels of stress. This is consistent with our hypothesis derived from the literature on mooddependant memory. Injury memories are emotional by nature. Mental (personal) context reinstatement instruction may have enhanced memory accessibility of the experienced stress, and information particularly associated with this emotion, like injury details. Further controlled laboratory studies may be needed to explore this interpretation.

Several methodological limitations should be underlined. First, the test of the effect of stress on the recall of central details had low power due to a small sample size. In addition, it was difficult to consider another method than self-reported stress measures with real victims testifying about a past event. Yet, self-reported internal states are not always accurate (Safer et al., 2007). Engelberg and Christianson (2000) successfully tested the effectiveness of the CI in improving the recall of emotional states. Consequently, using the mental context reinstatement in future research to help victims to retrieve their emotional state and, thus, answering self-reported emotions scale more accurately, could be interesting. Furthermore, the use of a median split to differentiate between the two stress levels may create two samples that are too similar on this dimension. However, the results observed on the recall of central details are rather in line with those observed in the literature and are in the direction of the existence of a sufficient difference between the two samples. A replication of this study with a larger sample and more extreme samples on the dimension of the stress level would allow for a more powerful test. Note also that $95 \%$ of our sample was composed of students, which may 
limit the generalization of our results mainly to the student population. Finally, as in most field studies, it was not possible to access the ground truth about what had happened. It should be noted, however, that there are a significant number of laboratory studies that have shown that $\mathrm{CI}$ is associated with an increase in quantity of details reported and with similar accuracy rates (i.e., increases in the number of correct and incorrect details recalled) compared to a control interview protocol (see Memon et al., 2010).

The present study highlights the practical relevance of the CI. First, very long retention delays (2-21 months) were included, which is rare in the CI literature (typically on the order of a few minutes or a few days). That the CI is beneficial for long delays is particularly important for many real-world investigations, which may extend over months or years (Fisher \& Geiselman, 2019; Amann, 2017). Second, the benefit observed with the CI was particularly large in our study: Victims interviewed with the CI reported 3 times more event-related details, 3 times more central information and 6 times more peripheral information. Third, the CI can be used in a stressful context. Its benefit on the recall of very central details (such as injuries) can even be amplified in high stress situations. We have not observed any contraindications to its use in a stressful context, even though studies on this topic are probably still lacking.

In conclusion, this study demonstrated the relevance of the CI to improve the longterm recall of information from actual victims of road accidents. We therefore encourage scholars to extend this kind of study to criminal events, such as robberies, physical and/or sexual violence, to non-criminal events such as oral history of dangerous events, military events, or genocide-related experiences (see Fisher \& Geiselman, 2019). We also encourage more research on long retention intervals. Evidence that witness accounts can be particularly complete despite very long intervals should be confirmed. In addition, more data are needed 
on the relationship between the length of these intervals and the accuracy of witness testimony collected through evidence-based interview protocols.

\section{References}

Amann, W. (2017). Cognitive interviewing brings cold case rapist to justice. Air Force Office of Special Investigations Public Affairs. March 27, 2017. Retrieved from: https://urldefense.proofpoint.com/v2/url?u=http-3A__www.osi.af.mil_News_Article2DDisplay_Article_1130739_cognitive-2Dinterviewing-2Dbrings-2Dcold-2Dcase2Drapist-2Dto-

2Djustice_\&d=DwIFAg\&c=lhMMI368wojMYNABHh1gQQ\&r=ypR12vpU5MrSJqa9 GyToYg\&m=MhlPSRWUD_zpt5o2Xyxv5Lg54wimzXy8iZFAFBfZijA\&s=HwYkPw KdPRe6U2b0rJfoUe6KpiYOXOIYpz9b0G34h5M\&e=

Beck, A. T., Steer, R. A., \& Garbin, M. G. (1988). Psychometric properties of the Beck Depression Inventory: Twenty-five years of evaluation. Clinical Psychology Review, 8(1), 77-100. https://doi-org.insb.bib.cnrs.fr/10.1016/0272-7358(88)90050-5

Bensi, L., Nori, R., Gambetti, E., \& Giusberti, F. (2011). The Enhanced Cognitive Interview: A study on the efficacy of shortened variants and single techniques. Journal of Cognitive Psychology, 23(3), 311-321. https://doiorg.insb.bib.cnrs.fr/10.1080/20445911.2011.497485

Berntsen, D. (2002). Tunnel memories for autobiographical events: Central details are remembered more frequently from shocking than from happy experiences. Memory \& Cognition, 30(7), 1010-1020. https://doi-org.insb.bib.cnrs.fr/10.3758/BF03194319

Christianson, S.-Å. (1992). Emotional stress and eyewitness memory: A critical review. Psychological Bulletin, 112(2), 284-309. https://doi-org.insb.bib.cnrs.fr/10.1037/00332909.112.2.284 
Colomb, C., Ginet, M., Wright, D., Demarchi, S., \& Sadler, C. (2013). Back to the real: Efficacy and perception of a modified cognitive interview in the field. Applied Cognitive Psychology, 27(5), 574-583.

Clifford, B. R., \& George, R. (1996). A field evaluation of training in three methods of witness/victim investigative interviewing. Psychology, Crime \& Law, 2(3), 231-248. https://doi-org.insb.bib.cnrs.fr/10.1080/10683169608409780

Engelberg, E., \& Christianson, S.-Å. (2000). Recall of unpleasant emotion using memoryenhancing principles. Psychology, Crime \& Law, 6(2), 99-112. https://doiorg.insb.bib.cnrs.fr/10.1080/10683160008410835

Eich, E. (1995). Mood as a mediator of place dependent memory. Journal of Experimental Psychology: General, 124(3), 293-308. https://doi-org.insb.bib.cnrs.fr/10.1037/00963445.124 .3 .293

Fisher, R. P. \& Geiselman, R. E. (1992). Memory-enhancing techniques in investigative interviewing: The cognitive interview. Springfield, IL: C.C. Thomas.

Fisher, R. P., \& Geiselman, R. E. (2019). Expanding the cognitive interview to non-criminal investigations. In J. Dickinson, N. Schreiber Compo, R. Carol, B. Schwartz, \& M. McCauley (Eds.). Evidence-based Investigative Interviewing: Applying Cognitive Principles (pp. 1-28). New York: Routledge: Taylor \& Francis.

Fisher, R. P., Geiselman, R. E., \& Amador, M. (1989). Field test of the cognitive interview: Enhancing the recollection of actual victims and witnesses of crime. Journal of Applied Psychology, 74(5), 722-727. https://doi-org.insb.bib.cnrs.fr/10.1037/00219010.74 .5 .722

Ginet, M., \& Verkampt, F. (2007). The cognitive interview: Is its benefit affected by the level of witness emotion? Memory, 15(4), 450-464. https://doiorg.insb.bib.cnrs.fr/10.1080/09658210601092670 
Holliday, R. E., Humphries, J. E., Milne, R., Memon, A., Houlder, L., Lyons, A., \& Bull, R. (2012). Reducing misinformation effects in older adults with cognitive interview mnemonics. Psychology and Aging, 27(4), 1191-1203. https://doiorg.insb.bib.cnrs.fr/10.1037/a0022031

Krix, A. C., Sauerland, M., Raymaekers, L. H. C., Memon, A., Quaedflieg, C. W. E. M., \& Smeets, T. (2016). Eyewitness evidence obtained with the Self-Administered Interview@ is unaffected by stress. Applied Cognitive Psychology, 30(1), 103-112. https://doi-org.insb.bib.cnrs.fr/10.1002/acp.3173

Memon, A., Meissner, C. A., \& Fraser, J. (2010). The cognitive interview: A meta-analytic review and study space analysis of the past 25 years. Psychology, Public Policy, \& Law, 16(4), 340-372. doi:10.1037/a0020518

Peace, K. A., \& Porter, S. (2004). A Longitudinal Investigation of the Reliability of Memories for Trauma and other Emotional Experiences. Applied Cognitive Psychology, 18(9), 1143-1159. https://doi-org.insb.bib.cnrs.fr/10.1002/acp.1046

Safer, M. A., Breslin, C. W., Boesch, R. P., \& Cerqueira, R. (2007). Long-term memory for the emotional gist and the emotional essence of an experience. Memory, 15(8), 861872. https://doi-org.insb.bib.cnrs.fr/10.1080/09658210701738412

Shields, G. S., Sazma, M. A., McCullough, A. M., \& Yonelinas, A. P. (2017). The effects of acute stress on episodic memory: A meta-analysis and integrative review. Psychological Bulletin, 143(6), 636-675. https://doi-org.insb.bib.cnrs.fr/10.1037/bu10000100

Sundin, E. C., \& Horowitz, M. J. (2002). Impact of Event Scale: Psychometric properties. The British Journal of Psychiatry, 180(3), 205-209. https://doiorg.insb.bib.cnrs.fr/10.1192/bjp.180.3.205 
Table 1. Means and standard deviations of event-related details, $d f, F$-values, $p$-values and effect sizes, as a function of the interview condition and the stress category

\begin{tabular}{|c|c|c|c|c|c|c|c|c|c|c|}
\hline & \multicolumn{5}{|c|}{ Interview condition } & \multicolumn{5}{|c|}{ Stress category } \\
\hline & $\mathrm{CI}(n=29)$ & $\begin{array}{l}\text { Control } \\
(n=27)\end{array}$ & $F(1,55)$ & $p$ & $\eta_{\mathrm{p}}^{2}$ & $\begin{array}{l}\text { High-stress } \\
(n=28)\end{array}$ & $\begin{array}{c}\text { Low-stress } \\
(n=28)\end{array}$ & $F(1,55)$ & $p$ & $\eta_{\mathrm{p}}^{2}$ \\
\hline Event-related details & $97.93(55.14)$ & $32.82(15.71)$ & 36.40 & $<.001$ & .412 & $77.79(59.68)$ & $55.29(41.98)$ & 3.39 & .071 & .061 \\
\hline Actions & $35.38(21.73)$ & $9.89(6.52)$ & 36.01 & $<.001$ & .405 & $26.71(24.54)$ & $19.46(15.41)$ & 2.23 & .141 & .038 \\
\hline People & $5.86(8.23)$ & $4.48(4.14)$ & 0.61 & .439 & .013 & $4.18(6.54)$ & $6.21(6.55)$ & 1.39 & .243 & .026 \\
\hline Location & $16.07(10.03)$ & $5.44(3.63)$ & 31.94 & $<.001$ & .333 & $12.89(10.83)$ & $9.00(7.08)$ & 2.47 & .122 & .045 \\
\hline Objects & $2.52(2.80)$ & $0.48(0.80)$ & 12.92 & $<.001$ & .198 & $1.68(2.38)$ & $1.39(2.28)$ & 0.14 & .708 & .002 \\
\hline $\begin{array}{l}\text { Words and } \\
\text { conversations }\end{array}$ & $7.00(10.38)$ & $0.82(1.14)$ & 9.71 & .003 & .153 & $5.61(10.59)$ & $2.43(3.90)$ & 2.23 & .142 & .039 \\
\hline Cars & $4.97(3.17)$ & $2.04(2.24)$ & 15.72 & $<.001$ & .229 & $3.96(3.54)$ & $3.14(2.61)$ & 0.94 & .3336 & .017 \\
\hline Injuries & $5.38(5.73)$ & $0.41(0.93)$ & 23.35 & $<.001$ & .310 & $4.50(6.20)$ & $1.46(2.12)$ & 8.07 & .006 & .127 \\
\hline Other & $20.40(16.80)$ & $9.07(8.69)$ & 9.99 & .003 & .017 & $12.00(12.40)$ & $17.90(16.10)$ & 2.30 & .135 & .006 \\
\hline
\end{tabular}


Table 2. Means and standard deviations of central and peripheral details, $d f, F$-values, $p$-values and effect sizes, as a function of the interview condition and the stress category

\begin{tabular}{lccccccccccccc}
\hline \multicolumn{1}{c}{ Interview condition } \\
\hline \multicolumn{1}{c}{$\begin{array}{c}\text { Control } \\
\end{array}$} & $\mathrm{CI}(n=29)$ & $(n=27)$ & $d f$ & $F$ & $p$ & $\eta_{\mathrm{p}}{ }^{2}$ & & $\begin{array}{c}\text { High-stress } \\
(n=28)\end{array}$ & $\begin{array}{c}\text { Low-stress } \\
(n=28)\end{array}$ & $F(1,55)$ & $p$ & $\eta_{\mathrm{p}}{ }^{2}$ \\
\hline Central & $88.97(55.87)$ & $27.41(17.00)$ & 1,55 & 32.74 & $<.001$ & .386 & & $71.68(61.45)$ & $46.89(37.18)$ & 4.24 & .050 & .075 \\
Peripheral & $66.00(51.03)$ & $10.15(9.35)$ & 1,55 & 31.78 & $<.001$ & .379 & & $48.25(54.37)$ & $29.89(35.62)$ & 2.66 & .109 & .049 \\
\hline
\end{tabular}

